\title{
NOTA
}

\section{VARIABILIDADE ESPACIAL DE ATRIBUTOS FÍSICOS DE UM LATOSSOLO VERMELHO DISTROFÉRRICO, SUBMETIDO A DIFERENTES MANEJOS ${ }^{(1)}$}

\author{
Welson Lima Simões ${ }^{(2)}$, Élio Lemos da Silva ${ }^{(3)}$, Daniela Miranda de \\ Lima $^{(4)} \&$ Marcelo Silva de Oliveira ${ }^{(5)}$
}

\begin{abstract}
RESUMO
O conhecimento da variabilidade espacial de atributos do solo serve de subsídio para a determinação de estratégias específicas de manejo que otimizem a produtividade agrícola. O objetivo deste trabalho foi avaliar a influência do manejo do solo na variabilidade espacial de alguns atributos físicos de um Latossolo Vermelho distroférrico. O estudo foi realizado, no ano de 2002, em três áreas situadas no município de Lavras (MG), submetidas a diferentes manejos em decorrência do uso atual do solo: A1 - área em pousio há três anos; A2 - área recentemente plantada com café e A3 - área cultivada com café há três anos. A amostragem foi disposta em malha com espaçamentos entre pontos de 1,5 e $10 \mathrm{~m}$, totalizando 188 pontos amostrados. Em cada ponto, foram coletadas amostras deformadas de solo, na camada de 0-20 cm, para avaliação dos teores de argila, silte, areia e da densidade de partículas. Esses dados foram analisados pela estatística descritiva (software Sisvar) e pela geoestatística (software GeoR). Concluiu-se que o manejo e a posição no terreno influenciaram a variabilidade espacial dos atributos estudados. Os maiores "alcances" dos semivariogramas foram encontrados para as áreas com maior revolvimento do solo. Em média, silte e densidade de partículas apresentaram os maiores e menores coeficientes de variação, respectivamente.
\end{abstract}

Termos de indexação: manejo do solo, geoestatistica, krigagem.

\footnotetext{
${ }^{(1)}$ Parte da Tese de Mestrado do primeiro autor, apresentada ao Departamento de Engenharia agrícola, Universidade Federal de Lavras - UFLA. Recebido para publicação em junho de 2005 e aprovado em outubro de 2006.

(2) Doutorando do Departamento de Engenharia Agrícola, Universidade Federal de Viçosa - UFV. CEP 36.570-000 Viçosa-(MG). Bolsista CNPq. E-mail: welsimoes@yahoo.com.br

(3) Professor do Departamento de Engenharia Agrícola, Universidade Federal de Lavras - UFLA. Caixa Postal 37 CEP 37.200-000 Lavras (MG). E-mail: elemos@ufla.br

${ }^{(4)}$ Doutoranda do Departamento de Solos, UFV. E-mail: danielamlim@yahoo.com.br

(5) Professor do Departamento de Ciências Exatas, UFLA. E-mail: marcelso@ufla.br
} 


\title{
SUMMARY: SPATIAL VARIABILITY OF PHYSICAL ATTRIBUTES OF A DYSTROFERRIC RED LATOSOL UNDER DIFFERENT MANAGEMENTS
}

\begin{abstract}
The study of the spatial variability of soil attributes can help in the determination of a specific management strategy that enhances agricultural productivity. The present study aimed at the evaluation of a possible influence of the soil management on spatial variability of a Dystroferric Red Latosol (Oxisol) physical properties. The study was carried out in 2002, in three adjacent areas, in Lavras, Minas Gerais state, Brazil, with the following management systems: A1 - fallowing for three years; A2 - recently planted coffee plants and $A 3$ - area with three-year-old coffee plants. One hundred and eighty-eight grid points were sampled at distances of 1,5 and $10 \mathrm{~m}$. Disturbed soil samples were collected at each sampling point in the $0-20 \mathrm{~cm}$ layer to evaluate the clay, silt, and sand contents and particle density. Data were analyzed by classical statistics (Sisvar software) and geostatistics (GeoR software). It was concluded that management and land position influenced the spatial variability of the studied attributes, and the widest range of semivariograms was found in the areas with greatest soil disturbance. On average, silt and particle density presented the largest and smallest variation coefficients, respectively.
\end{abstract}

Index terms: soil management, geostatistics, kriging.

\section{INTRODUÇÃO}

Os atributos físicos e químicos do solo influenciam diretamente o crescimento e o desenvolvimento das culturas. Desta forma, a avaliação da variabilidade espacial destes atributos tem-se tornado importante ferramenta na determinação de estratégias de manejo do solo, que procuram aumentar a produtividade agrícola (Trangmar et al., 1985).

Segundo Silveira \& Cunha (2002), numa área cultivada, além da variabilidade natural, existem fontes adicionais de heterogeneidade no solo, por causa do manejo exercido pelo homem das mais variadas formas. Tais variações, mais pronunciadas nos sistemas convencionais de preparo do solo do que nos conservacionistas, influenciam principalmente o acúmulo de material orgânico, a infiltração da água no solo e a erosão hídrica (Bertol et al., 2004).

Dentre os diversos fatores que influem na variabilidade espacial das propriedades físicas e químicas do solo, Souza (2001) cita a posição do terreno na paisagem (declividade e forma do relevo) como uma característica de grande influência. O autor acrescenta que a forma da paisagem pode gerar rotas preferenciais para o fluxo de água, podendo afetar a variabilidade espacial das propriedades do solo.

A análise da variabilidade de atributos físicos do solo pode ser realizada por meio da estatística descritiva. Esta ferramenta, entretanto, não considera a distribuição dos dados no espaço, ao contrário da geoestatistica, que considera a dependência espacial entre as amostras e a sua localização geográfica (Vieira, 2000).
Em áreas muito grandes ou heterogêneas, a amostragem de todos os pontos no solo, dos quais se deseja obter informações, é, na maioria das vezes, uma opção cara e trabalhosa. A análise geoestatística possibilita a construção de variogramas que servem de suporte para a técnica da krigagem, permitindo a representação quantitativa da variação de um fenômeno regionalizado no espaço (Huijbregts, 1975). Com o procedimento geoestatístico da krigagem, portanto, é possível estimar valores de pontos não amostrados no solo, sem tendência e com variância mínima (Vieira, 2000).

Hamlett et al. (1986) afirmam que os dados devem preencher certas condições de estacionaridade para que o semivariograma possa ser construído e interpretado. Segundo Vieira (2000), a hipótese menos restritiva para uso da geoestatística é a hipótese intrínseca, segundo a qual a função do semivariograma existe e a variância das diferenças depende apenas da distância entre as amostras. Em situações reais, no entanto, problemas, como a existência de tendência nos dados, podem influir nas análises e devem ser corrigidos para evitar falsas conclusões. Uma das alternativas para retirada da tendência dos dados é o procedimento descrito por Davis (1986), segundo o qual se pode ajustar um polinômio de primeiro ou segundo grau, para os valores da propriedade, de acordo com as coordenadas, e fazer com que o resíduo seja obtido pela diferença entre valor medido e valor do polinômio em cada ponto.

Segundo Vieira (2000), numa análise geoestatística, os valores de alcance dos semivariogramas experimentais informam, ainda, as distâncias 
mínimas entre amostras, para serem consideradas independentes. A independência ou não dos pontos amostrados é fundamental para escolha do teste estatístico a ser aplicado.

O presente trabalho foi desenvolvido com o objetivo de avaliar a variabilidade espacial de atributos físicos de um Latossolo Vermelho distroférrico, submetido a diferentes manejos, por meio da estatística descritiva e da geoestatística.

\section{MATERIAL E MÉTODOS}

O presente estudo foi desenvolvido no município de Lavras (MG), a $21^{\circ} 14$ ' de latitude Sul e a $45^{\circ} 00$ ' de longitude Oeste de Greenwich, com altitude média de 918 m, ao sul do Estado de Minas Gerais, no ano de 2002. O solo da área estudada é o Latossolo Vermelho distroférrico, segundo classificação da Embrapa (1999). Segundo a classificação do Departamento de Agricultura dos EUA (USDA), os solos das áreas 1 e 2 são classificados como argilosos e, da área 3, como muito argiloso (Quadro 1). A diferença de textura do solo entre as áreas, apesar de pequenas, pode estar relacionada com a sua posição topográfica, de forma que a área 3 , por estar localizada na parte mais baixa do terreno e ter uma forma côncava, recebia partículas mais finas transportadas pela água, por erosão hídrica, das partes mais altas.

Foram avaliadas três áreas vizinhas, com dimensões de $20 \times 100 \mathrm{~m}$ cada, considerando as direções leste-oeste e norte-sul, respectivamente, que apresentavam as seguintes características: área 1 localizada na parte superior do terreno, com 1,07\% de declividade na direção sul-norte e $6,03 \%$ na direção leste-oeste. Esta área encontrava-se em pousio durante os três últimos anos. A última intervenção no solo desta área foi uma aração e uma gradagem, nas profundidades de 0,4 e $0,2 \mathrm{~m}$, respectivamente, porém, sem instalação de cultura; área 2 - situada na parte intermediária do terreno, com 1,9\% de declividade na direção sul-norte e 7,6 \% na direção leste-oeste, foi plantada com café, dois meses antes do início da coleta de dados, com o seguinte manejo: uma aração, uma gradagem e um sulcamento, nas profundidades de 0,4 , 0,2 e $0,4 \mathrm{~m}$, respectivamente, e duas passagens de trator com carreta, para adubação e plantio das mudas, e área 3 - situada na parte inferior do terreno, com $0,72 \%$ de declividade na direção sul-norte e 11,37\% na direção leste-oeste, a qual se encontra plantada com café há três anos, manejada com colheita manual. $\mathrm{O}$ único trato cultural mecanizado era a passagem de enxada rotativa nas entrelinhas, uma vez por ano. Antes da instalação do experimento, as três áreas faziam parte de um cafezal abandonado.

A amostragem do solo foi efetuada em forma de malha retangular, com espaçamentos variando, em geral, de 5 a $10 \mathrm{~m}$. Em algumas seções, esse espaçamento foi reduzido para $1 \mathrm{~m}$ (Figura 1), com o objetivo de minimizar o efeito pepita dos semivariogramas, conforme recomendação de Ribeiro Junior (1995). No total, foram coletadas 188 amostras e, em cada ponto, foram feitas amostragens na camada de $0-0,2 \mathrm{~m}$.

A densidade de partículas foi determinada pelo Método do Balão Volumétrico, e a análise granulométrica

Quadro 1. Estatística descritiva, referente aos teores de areia, silte, argila e densidade de partículas do solo, para as três áreas estudadas

\begin{tabular}{|c|c|c|c|c|c|c|c|c|c|c|c|c|}
\hline \multirow{2}{*}{$\begin{array}{l}\text { Parâmetro } \\
\text { estatístico }\end{array}$} & \multicolumn{3}{|c|}{ Areia } & \multicolumn{3}{|c|}{ Silte } & \multicolumn{3}{|c|}{ Argila } & \multicolumn{3}{|c|}{ Densidade de partícula } \\
\hline & A1 & A2 & A3 & A1 & A2 & A3 & A1 & A2 & A3 & A1 & A2 & A3 \\
\hline Número de pontos & 63 & 63 & 62 & 63 & 63 & 62 & 63 & 63 & 62 & 63 & 63 & 62 \\
\hline Valores médios & 16,63 & 18,66 & 17,83 & 23,48 & 23,30 & 19,51 & 59,89 & 58,04 & 62,65 & 2,663 & 2,660 & 2,651 \\
\hline Variância & 11,35 & 22,89 & 14,30 & 39,38 & 20,81 & 34,97 & 54,42 & 37,63 & 35,13 & 0,003 & 0,005 & 0,005 \\
\hline Desvio-padrão & 3,369 & 4,785 & 3,781 & 6,275 & 4,561 & 5,914 & 7,377 & 6,135 & 5,927 & 0,057 & 0,070 & 0,068 \\
\hline CV (\%) & 20,26 & 25,65 & 21,20 & 26,72 & 19,58 & 30,31 & 12,32 & 10,57 & 9,459 & 2,131 & 2,634 & 2,561 \\
\hline Assimetria & 0,984 & 0,745 & 0,940 & 0,350 & $-0,446$ & 0,366 & $-0,240$ & $-0,744$ & $-0,595$ & $-0,447$ & $-0,179$ & $-0,072$ \\
\hline Curtose & 2,758 & 2,783 & 2,748 & 2,025 & 3,752 & 2,794 & 2,224 & 2,632 & 2,425 & 2,867 & 2,154 & 1,751 \\
\hline Mínimo & 12,50 & 12,07 & 13,12 & 12,42 & 11,80 & 7,732 & 44,35 & 41,73 & 48,47 & 2,530 & 2,500 & 2,530 \\
\hline Máximo & 24,94 & 31,92 & 27,40 & 37,39 & 34,48 & 33,49 & 74,14 & 66,21 & 71,70 & 2,780 & 2,780 & 2,780 \\
\hline Amplitude total & 12,44 & 19,85 & 14,27 & 24,97 & 22,68 & 25,76 & 29,79 & 24,48 & 23,24 & 0,250 & 0,280 & 0,250 \\
\hline Mediana & 15,99 & 17,76 & 17,05 & 22,41 & 23,74 & 18,89 & 60,40 & 59,39 & 63,92 & 2,669 & 2,668 & 2,659 \\
\hline Moda & 15,34 & 16,51 & 16,42 & 20,33 & 23,92 & 18,23 & 62,22 & 60,96 & 65,31 & 2,679 & 2,722 & 2,705 \\
\hline
\end{tabular}

CV: coeficiente de variação; teores médios de areia, silte e argila em (\%) e densidade de partículas em (g cm³); A1 - área 1 ; A2 área 2 e A3 - área 3. 




Figura 1. Croqui da área experimental e respectivo esquema de amostragem, com unidades em metros. A1, A2 e A3 são as áreas 1, 2 e 3 respectivamente. NM é o norte magnético.

das amostras, pelo Método da Pipeta, descrito por Day (1965). Os dados foram analisados por meio da estatística descritiva, com uso do software Sisvar (2000), e da geoestatística, com uso do software GeoR (2003).

Para fins de comparação, foram adotados os limites de coeficiente de variação (CV) propostos por Warrick \& Nielsen (1980), para a classificação da variabilidade dos atributos analisados, sendo: CV $<12 \%$, $12 \%<\mathrm{CV}<60 \%$ e CV $>60 \%$, considerados de baixa, média e alta variabilidade, respectivamente.

A dependência espacial foi analisada por meio de semivariogramas, cuja representação dos modelos é feita por meio da semivariância em função da distância $\gamma(\mathrm{h})$, conforme Equacão (1).

$$
\gamma^{*}(h)=\frac{1}{2 N(h)} \sum_{i=1}^{N(h)}\left[Z\left(x_{i}\right)-Z\left(x_{i}+h\right)\right]^{2}
$$

em que $N(h)$ é o número de pares de valores medidos, $z\left(x_{i}\right)$ e $z\left(x_{i}+h\right)$, valores separados por um vetor de distância h.

A escolha do melhor modelo foi feita por meio da autovalidação, seguindo método de Vieira (2000), no qual se selecionam os modelos com a média do erro mais próxima de zero e a variância do erro mais próxima de 1. Após a escolha do modelo, foram obtidos os parâmetros: alcance, contribuição e efeito pepita.
O grau de dependência espacial (GD), que determina quanto da variância espacial está presente na variância total e, conseqüentemente, dá idéia da confiança que se pode ter nas estimativas (Vieira, 2000), foi calculado por meio da equação (2), cuja classificação, segundo Cambardella et al. (1994), é de forte, para GD $=25 \%$; moderado, para GD entre $25 \mathrm{e}$ $75 \%$, e fraco, para GD > $75 \%$ :

$$
G D=\frac{C_{0}}{C_{0}+C_{1}} * 100
$$

em que $\mathrm{C}_{0}$ é o efeito pepita e $\mathrm{C}_{1}$ a contribuição.

Nos semivariogramas, considerou-se a isotropia, uma vez que, por meio da autovalidação, observou-se uma pequena contribuição à exatidão das estimações, quando considerada a anisotropia. O método de interpolação utilizado foi a krigagem ordinária, efetuada por meio do programa Surfer (1999).

\section{RESULTADOS E DISCUSSÃO}

Os valores de média, mediana e moda, para areia, foram próximos entre si, porém, demonstraram certa assimetria à direita na distribuição dos valores, nas três áreas estudadas (Quadro 1). Os dados apresentaram comportamento próximo da distribuição normal, com coeficiente de curtose próximo de 3 e coeficiente de assimetria afastando-se pouco de zero. A normalidade para este atributo também foi encontrada por Gonçalves \& Folegatti (1995), trabalhando com Terra Roxa Estruturada (Nitossolo Vermelho). De acordo com a classificação proposta por Warrick \& Nielsen (1980), a areia apresentou média variabilidade, nas três áreas estudadas. Os valores de CV encontrados neste trabalho mostraram-se menores que os observados por Correa (1986), para a mesma classe de solo estudada (38,9 e 39,6 \%), e maiores que os observados por Silva (1988), em solo argiloso (11,5\%).

Segundo a classificação proposta por Warrick \& Nielsen (1980), o silte apresentou média variabilidade para as três áreas, porém, no geral, os valores de CV encontrados foram maiores que para areia. Esta maior variabilidade, para silte, pode estar relacionada com a sua maior mobilidade no solo. Além disso, o seu processo de determinação, que pode ter incorporado parte da variabilidade existente nos atributos areia e argila, pode ter contribuído para essa maior variabilidade. Este fato também foi observado por Roque (2003) e Silva (1988). Os valores de CV encontrados para silte foram menores do que os citados por Correa (1986), para a mesma classe de solo (67,4 e $44 \%)$ e próximos aos encontrados por Silva (1988), para solo argiloso (26\%). As áreas 1 e 3 apresentaram assimetria à direita e a área 2 uma ligeira assimetria à esquerda, para silte, todas com um comportamento próximo da distribuição normal. A normalidade para silte também foi encontrada por Gonçalves \& Folegatti (1995), trabalhando com Terra Roxa Estruturada. 
Segundo a classificação proposta por Warrick \& Nielsen (1980), na área 1, o atributo argila apresentou média variabilidade e, nas áreas 2 e 3 , baixa variabilidade (Quadro 1). No caso da área 1, sua posição no relevo e o maior processo de translocação destas partículas em áreas pouco revolvidas (Carvalho et al., 1999) podem ter influenciado a variabilidade. A influência da posição no relevo sobre a distribuição de argila também foi citada por Pocay (2000). Os valores de CV encontrados neste trabalho foram menores do que os observados por Correa (1986), para esta mesma classe de solo (18,4 e 15,5\%), e maiores que os observados por Silva (1988), para solo argiloso (5\%). Observa-se para argila, uma pequena assimetria à esquerda na distribuição dos valores, para as três áreas estudadas (Quadro 1). Os dados demonstram comportamento próximo da distribuição normal para as três áreas. A normalidade para este atributo também foi encontrada por Gonçalves \& Folegatti (1995), trabalhando com Terra Roxa Estruturada.

Os valores de densidade de partículas (Dp) demonstraram pequena assimetria à esquerda, nas três áreas estudadas (Quadro 1). Observaram-se normalidade para os valores das áreas 1 e 2, e nãonormalidade, para os da área 3 , esta última confirmada pelo teste de Shapiro-Wilk. A nãonormalidade também foi observada por Silva (1988) para este atributo. A falta de normalidade para Dp na área 3 pode estar relacionada com a deposição de materiais carreados pela chuva, de áreas localizadas acima no relevo, proporcionando o surgimento de subáreas com dados atípicos. Segundo Kiehl (1979), a Dp está relacionada também com os teores de matéria orgânica no solo e, sendo a área 3 caracterizada por apresentar maior quantidade de plantas de grande porte, a densidade de raízes pode ter influenciado os resultados. Segundo a classificação proposta por Warrick \& Nielsen (1980), a Dp é de baixa variabilidade, para as três áreas. Os valores de CV encontrados estão próximos dos citados por Correa (1986), para a mesma classe de solo (5,5 e 4,1\%).
A variabilidade espacial apresentou, no geral, estrutura de dependência espacial para todos os atributos do solo estudados. Para areia, verificou-se tendência de aumento no sentido sul-norte, nas três áreas, a qual foi retirada por meio de rotinas do programa GeoR, utilizando um modelo de primeiro grau. Segundo Ribeiro Junior (1995), essa tendência é incompatível com a hipótese intrínseca e a nãocorreção dessa tendência leva a interpretações inadequadas que comprometem e talvez invalidem toda a análise. O modelo de semivariograma ajustado para areia, nas áreas 1 e 2 , foi o Gaussiano e, na área 3 , o Esférico. A área 3 apresentou valor de alcance muito superior ao maior comprimento da área amostrada. Neste caso, seria recomendável amostrar o solo a distâncias maiores, para obter os verdadeiros valores de alcance e contribuição. Segundo a classificação proposta por Cambardella et al. (1994), para as áreas 1 e 2, o grau de dependência (GD) é moderado e, para a área 3 , forte. Segundo estes autores, variáveis que apresentam forte grau de dependência (GD) são mais influenciadas por propriedades intrínsecas do solo. Os valores de alcance observados na literatura, para areia, são menores que os encontrados neste trabalho. Gonçalves \& Folegatti (1995), para Terra Roxa Estruturada, encontraram $30 \mathrm{~m}$ e Libardi et al. (1986), também para Terra Roxa Estruturada, encontraram $10 \mathrm{~m}$. Esta diferença de valores de alcance para o mesmo solo pode, também, ser um indicativo da influência do manejo na variabilidade espacial deste atributo.

Para silte, na área 2 , também foi observada e retirada tendência nos dados. Esta tendência de decréscimo no sentido norte-sul pode estar relacionada com a maior declividade dessa área, quando comparada com as demais, o que facilita o transporte do silte por erosão hídrica. O modelo de semivariograma ajustado para silte foi o Gaussiano e o maior alcance obtido foi para área 3, seguida das áreas 2 e 1, semelhantemente ao observado para areia (Quadro 2). Segundo classificação proposta por Cambardella et al. (1994), o GD é moderado nas três áreas. A variação dos valores

Quadro 2. Parâmetros dos semivariogramas ajustados para os teores de areia, silte, argila e densidade de partículas do solo, para as três áreas estudadas

\begin{tabular}{|c|c|c|c|c|c|c|c|c|c|c|c|c|}
\hline \multirow{2}{*}{$\begin{array}{l}\text { Parâmetro } \\
\text { estatístico }\end{array}$} & \multicolumn{3}{|c|}{ Areia } & \multicolumn{3}{|c|}{ Silte } & \multicolumn{3}{|c|}{ Argila } & \multicolumn{3}{|c|}{ Densidade de partícula } \\
\hline & A1 & A2 & A3 & A1 & A2 & A3 & A1 & A2 & A3 & A1 & A2 & A3 \\
\hline Modelo & Gaus ${ }^{\text {rt }}$ & Gaus ${ }^{\mathrm{rt}}$ & Esf $f^{r t}$ & Gaus & Gaus $^{\text {rt }}$ & Gaus & Gaus $^{\mathrm{rt}}$ & Gaus & Gaus $^{\mathrm{rt}}$ & Gaus & Gaus & * \\
\hline $\mathrm{C}_{0}$ & 1,017 & 5,835 & 1,865 & 23,15 & 12,69 & 25,94 & 28,02 & 25,00 & 23,04 & 0,003 & 0,004 & * \\
\hline $\mathrm{C}_{1}$ & 2,139 & 6,878 & 13,44 & 23,71 & 8,598 & 23,54 & 22,37 & 46,30 & 12,71 & 0,001 & 0,686 & * \\
\hline A & 34,48 & 38,99 & 295,2 & 25,87 & 59,04 & 73,36 & 25,79 & 106,6 & 64,11 & 71,47 & 1373,6 & * \\
\hline GD & 32,22 & 45,90 & 12,19 & 49,40 & 59,60 & 52,42 & 55,61 & 35,06 & 64,45 & 74,36 & 0,64 & $*$ \\
\hline
\end{tabular}

$\mathrm{C}_{0}$ - efeito pepita; $\mathrm{C}_{1}$ - contribuição; A - alcance $(\mathrm{m}) ; \mathrm{GD}$ - grau de dependência (\%); * dados sem estrutura de dependência espacial; Esf - esférico; Gaus - gaussiano; ${ }^{\text {rt }}$ - retirado à tendência; A1 - área 1; A2 - área 2 e A3 - área 3. 
de alcance entre as áreas, para silte, não corrobora o observado por Guimarães (2000), que encontrou o mesmo valor de alcance em dois sistemas de manejo distintos (40 m).

Para argila, observou-se tendência de decréscimo dos dados no sentido sul-norte, para as áreas 1 e 3, a qual também foi corrigida. O modelo de semivariograma ajustado para argila nas três áreas foi o Gaussiano, com maior alcance observado para área 2, seguida das áreas 3 e 1 (Quadro 2). Segundo classificação de Cambardella et al. (1994), as três áreas apresentaram GD moderado. Os alcances encontrados para argila, neste trabalho, mostraram-se superiores aos observados na literatura. Gonçalves \& Folegatti (1995), para Terra Roxa Estruturada, encontraram alcance de 30 m e Libardi et al. (1986), também para esse solo, encontraram $15 \mathrm{~m}$.

Para a densidade de partículas, apenas a área 3 não apresentou estrutura de dependência espacial entre os dados amostrados, sendo considerado efeito pepita puro. Isto indica que o valor do alcance deste atributo pode ser menor que o menor espaçamento entre amostras. Segundo Silva (1988), dados com estas características têm distribuição completamente aleatória, e a única estatística aplicável é a clássica, que considera independência entre as amostras. A justificativa para esse fato pode ser a mesma dada para falta de normalidade nos dados deste atributo. O modelo de semivariograma ajustado, para densidade de partículas das áreas 1 e 2, foi o Gaussiano, tendo a área 2 apresentado maior alcance e menor GD (Quadro 2). Para área 1, o GD foi moderado e, para área 2, forte, segundo classificação proposta por Cambardella et al. (1994). Com relação ao grande alcance observado na área 2 , foram seguidas as mesmas recomendações para areia na área 3. Grandes variações nos valores de alcance para este atributo também foram observadas por Roque (2003), em diferentes solos de uma transeção perpendicular a um dreno natural.
Numa análise geral da variabilidade espacial dos atributos estudados, observa-se que, apesar de serem áreas adjacentes com o mesmo solo e características pedológicas aparentemente homogêneas, a variação no manejo e na posição da paisagem alterou os parâmetros dos semivariogramas gerados. Este fato chama a atenção quanto ao cuidado que se deve ter ao utilizar parâmetros de semivariogramas gerados para outras áreas com características muito diferentes de manejo e composição paisagística, para escolha de espaçamento de amostragem, dentre outros usos.

Para todos os atributos do solo estudados, em especial os mais móveis (silte e argila), os maiores alcances foram observados nas áreas mais revolvidas (A2 e A3). Este fato pode estar relacionado com a maior homogeneização da camada onde foram coletadas as amostras (0-20 cm), como observado por Silveira \& Cunha (2002), após revolvimento da camada por máquinas. Neste caso, haveria homogeneização de possíveis depósitos dessas partículas, translocadas por rotas preferenciais nos sistemas em pousio ou de semeadura direta, após a aração e gradagem. Esta técnica de manejo, por sua vez, pode estar acarretando um comportamento espacial no sentido do tráfego dos implementos. Souza (1992) também observou maior variabilidade para alguns atributos do solo em sistemas de semeadura direta, quando comparado com o preparo convencional, e atribuiu este fato ao menor revolvimento do solo na semeadura direta.

Apesar de a estatística descritiva inferir sobre a variabilidade e sobre a condição média da área, não se sabe como esta variabilidade está distribuída no campo. As informações oferecidas pelos mapas de krigagem (Figuras 2 e 3) são mais completas que a simples apresentação dos semivariogramas, visto que, para cada área e para cada atributo, a variabilidade tem distribuição espacial diferenciada, como pode ser observado por meio de análise das isolinhas.

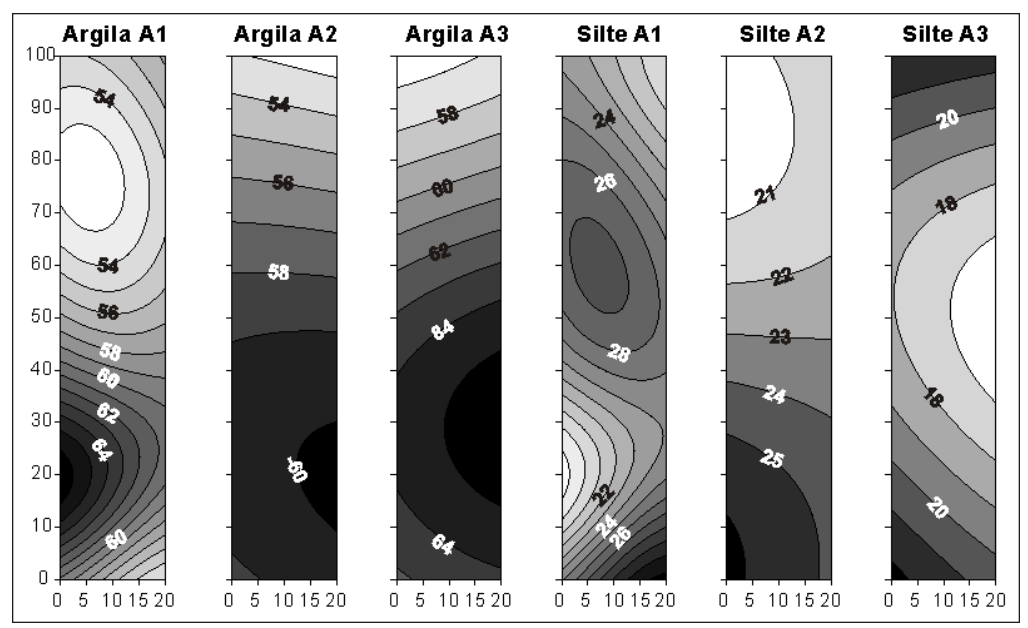

Figura 2. Mapeamento das áreas A1, A2 e A3, realizado por meio da krigagem, para os teores de argila e silte do solo. 




Figura 3. Mapeamento das áreas A1, A2 e A3, realizado por meio da krigagem, para os teores de areia e densidade de partículas (Dp) do solo.

A partir dos mapas apresentados, torna-se possível a separação das áreas em parcelas uniformes, ainda na fase de elaboração de projetos agronômicos ou em projetos já instalados, o que permite um planejamento adequado do manejo do solo e das culturas a serem instaladas nas áreas.

\section{CONCLUSÕES}

1. O manejo do solo e a posição da área na toposeqüência do terreno influenciam a variabilidade espacial dos teores de areia, silte e argila e da densidade de partículas do solo.

2. Os maiores alcances dos semivariogramas ocorrem nas áreas onde o solo sofre maior revolvimento mecânico.

3. O silte e a densidade de partículas do solo apresentam os maiores e menores coeficientes de variação, respectivamente.

4. O manejo do solo pode acarretar ausência de dependência espacial para densidade de partículas.

\section{LITERATURA CITADA}

BERTOL, I.; ALBUQUERQUE, J.A.; LEITE, D.; AMARAL, A.J. \& ZOLDAN Jr., W.A. Propriedades físicas do solo sob preparo convencional e semeadura direta em rotação e sucessão de culturas, comparadas às do campo nativo $\mathrm{R}$. Bras. Ci. Solo, 28:155-163, 2004.

CAMBARDELLA, C.A.; MOORMAN, T.B.; NOVAK, J.M.; PARKIN, T.B.; KARLEN, D.L.; TURCO, R.F. \& KONOPKA, A.E. Field-scale variability of soil properties in Central lowa Soils. Soil Sci. Soc. Am. J., 58:1501-1511, 1994.
CARVALHO, E.J.M.; FIGUEIREDO, M.S. \& COSTA, L.M. Comportamento fisico-hídrico de um Podzólico VermelhoAmarelo câmbico fase terraço sob diferentes sistemas de manejo, Brasília: Pesq. Agropec. Bras., 34:257-265, 1999.

CORREA, J.B.D. Variabilidade espacial de características e propriedades físicas de Latossolo Roxo do município de Lavras - MG. Lavras, Universidade Federal de Lavras, 1986. 83p. (Tese de Mestrado)

DAVIS, J.C. Statistics and data analysis in geology. 2.ed. New York, John Wiley, 1986, 646p.

DAY, P.R. Particle fractionation and particle size analysis. In: BLACK, C.A., ed.. Methods of soil analysis: Physical and mineralogical properties, including statistics of measurement and sampling. Madison, American Society of Agronomy, 1965. p.545-567.

EMPRESA BRASILEIRA DE PESQUISA AGROPECUÁRIA EMBRAPA. Centro Nacional de Pesquisas de Solos. Sistema Brasileiro de Classificação de Solos. Rio de Janeiro, 1999. 412p.

GEOR, The geoR package. Disponível em: www.est.ufpr.br/ geoR. Acesso, 29 set 2003.

GONÇALVES, A.C. \& FOLEGATTI, M.V. Variabilidade espacial de propriedades físicas do solo, ao longo de uma transeção em área irrigada por pivô central. In: CONGRESSO BRASILEIRO DE ENGENHARIA AGRÍCOLA, 24., Viçosa, 1995. Anais. Viçosa, Universidade Federal de Viçosa, 1995. v.1. p.190. CD-ROOM

GUIMARÃES, E.C. Variabilidade espacial de atributos de um Latossolo Vermelho Escuro textura argilosa da região do cerrado, submetido ao plantio direto e ao plantio convencional. Campinas, Universidade Estadual de Campinas, 2000. 85p. (Tese de Doutorado) 
HAMLETT, J.M.; HORTON, R. \& CRESSIE, N.A.C. Resistant and exploratory techniques for use in semivariogram analyses. Soil Sci. Soc. Am. J., 50:868-875, 1986.

HUIJBREGTS, C.J. Regionalized variables and quantitative analysis of spatial data. In: DAVIS, J.C. \& MCCULLAGH, M.J. eds. Display and analysis of spatial data. New York, John Wiley, 1975. p.38-53.

KIEHL, E.J. Manual de edafologia. São Paulo, Agronômica Ceres, 1979. 262p.

LIBARDI, P.L.; PREVEDELLO, C.L.; PAULETO, E.A. \& MORAES S.O. Variabilidade Espacial da umidade, textura e densidade de partículas ao longo de uma transeção, R. Bras. Ci. Solo, 10:85-90, 1986.

POCAY, V.G. Relações entre pedoforma e variabilidade espacial de atributos de Latossolos sob cultivo de cana-de-açúcar. Jaboticabal, Universidade Estadual Paulista, 2000. 135p. (Tese de Mestrado)

RIBEIRO JÚNIOR, P.J. Métodos geoestatísticos no estudo da variabilidade espacial de parâmetros do solo. Piracicaba, Escola Supeior “Luiz de Queiroz”, 1995. 99p. (Tese de Mestrado)

ROQUE, M.W. Variabilidade espacial de atributos físicohídricos em solos de uma transeção perpendicular a um dreno natural. Lavras, Universidade Federal de Lavras, 2003. 138p. (Tese de Mestrado)

SILVA, A.P. Variabilidade espacial de atributos físicos do solo. Piracicaba, Escola Supeior "Luiz de Queiroz", 1988. 105p. (Tese de Doutorado)
SILVEIRA, P.M. \& CUNHA, A.A. Variabilidade de micronutrientes, matéria orgânica e argila de um Latossolo submetido a sistemas de preparo. Brasília: Pesq. Agropec. Bras., 37:1325-1332, 2002.

SISVAR, Sistema para análise de variância, para Windows versão 4.3. Lavras, Universidade Federal de Lavras, 2000.

SOUZA, C.K. Relação solo-paisagem-erosão e variabilidade espacial de Latossolos em áreas sob cultivo de cana-deaçúcar no município de Jaboticabal (SP). Jaboticabal, Universidade Estadual Paulista, 2001. 186p. (Tese de Mestrado)

SOUZA, S.L. Variabilidade espacial do solo em sistema de manejo. Porto Alegre, Universidade Federaldo Rio Grande do Sul, 1992. 162p. (Tese de Doutorado)

SURFER, Surfer 7.0. Contouring and 3D surface mapping for scientist's engineers. User's Guide. New York: Golden software, Inc, 1999. 619p.

TRANGMAR, B.B.; YOST, R.S.; WADE, M.K. \& UEHARA, G. Applications of geostatistics to spatial studies of soil properties. Adv. Agron., 38:45-94, 1985.

VIEIRA, S.R. Geoestatística em estudo de variabilidade espacial do solo: In NOVAIS, R.F.; ALVAREZ V., V.H. \& SCHAEFER, C.E.G.R. Tópicos em ciência do solo. Viçosa, Sociedade Brasileira de Ciência do Solo, 2000. v.1, p. 1-55.

WARRICK, A.W. \& NIELSEN, D.R. Spatial variability of soil physical properties in the field. In: HILLEL, D., ed. Applications of soil physics. New York, Academic Press, 1980. 350p. 